\title{
Effects of extruder die head temperature and pre-gelatinized taro and broken rice flour level on physical properties of floating fish pellets
}

\begin{abstract}
Two experiments were conducted to investigate the effects of pre-gelatinized (PG) taro and broken rice and extruder die temperatures on the physical properties of extruded pellets. The first experiment was conducted to evaluate the effects of PG taro and extruder die head temperature $\left(125,140,155\right.$ and $\left.170{ }^{\circ} \mathrm{C}\right)$ and a subsequent experiment was conducted using PG broken rice instead. All the blends were preconditioned to a $40 \%$ moisture content and then extruded using a single screw extruder. The three zones of the barrel temperature profile $\left(70,90\right.$ and $\left.100^{\circ} \mathrm{C}\right)$ and screw speed $(150 \mathrm{rpm})$ of the extruder were constant throughout the extrusion cooking process. The physical properties of the pellets included floatability, expansion ratio, bulk density, pellet durability, water absorption and solubility, moisture content and pellet microstructure. The findings showed in both experiments that PG taro and broken rice inclusion levels and die temperature had significant effects on most of the physical properties of the pellets except for pellet durability index. Changing the inclusion rate of PG taro and broken rice from 15 to $25 \%$ significantly increased the expansion ratio and floatability of the extruded pellets. Similarly, as the die temperature was elevated in both experiment, the floatability of the extruded pellets in diet containing PG taro and broken rice increased by $114.62 \%$ and $21.88 \%$, respectively. It was also noted that use of PG taro and broken rice resulted in highly durable pellets in all treatments. Further, microstructure analysis of the extruded pellets revealed that using PG taro and broken rice, the surface of the extruded pellets became coarser when the die temperature was elevated from 125 to $170{ }^{\circ} \mathrm{C}$ and the PG taro and broken rice inclusion level was at $15 \%$. In conclusion, pre-gelatinized taro and broken rice could be used to manufacture higher quality floating pellets.
\end{abstract}

Keyword: Extrusion; Modified starch; Pre-gelatinized; Taro; Broken rice; Physical properties 